University of Wollongong

Research Online

Faculty of Engineering - Papers (Archive)

Faculty of Engineering and Information

Sciences

2009

\title{
Vertical and Radial Consolidation Analysis of Multilayered Soil Using the Spectral Method
}

\author{
Rohan T. Walker \\ University of Wollongong, rohanw@uow.edu.au \\ Buddhima Indraratna \\ University of Wollongong, indra@uow.edu.au \\ Nagaratnam Sivakugan \\ James Cook University Townsville
}

Follow this and additional works at: https://ro.uow.edu.au/engpapers

Part of the Engineering Commons

https://ro.uow.edu.au/engpapers/5215

\section{Recommended Citation}

Walker, Rohan T.; Indraratna, Buddhima; and Sivakugan, Nagaratnam: Vertical and Radial Consolidation Analysis of Multilayered Soil Using the Spectral Method, J. Geotech. Geoenviron. Eng: 135(5) 2009, 657-663.

https://ro.uow.edu.au/engpapers/5215

Research Online is the open access institutional repository for the University of Wollongong. For further information contact the UOW Library: research-pubs@uow.edu.au 


\title{
VERTICAL AND RADIAL CONSOLIDATION ANALYSIS OF \\ MULTI-LAYERED SOIL USING THE SPECTRAL METHOD
}

\author{
Rohan Walker \\ $\mathrm{PhD}$ (Wollongong) \\ Geotechnical Engineer, Coffey Geotechnics Pty Ltd, Sydney, Australia \& \\ Research Associate, Centre for Geomechanics and Railway Engineering, Faculty \\ of Engineering, University of Wollongong, Australia.
}

Buddhima Indraratna

$\mathrm{PhD}$, MSc (Lond), DIC, FASCE, FIEAust, FGS

Professor of Civil Engineering, Faculty of Engineering, University of Wollongong, Wollongong City, NSW 2522, Australia

Nagaratnam Sivakugan

MSCE (Purdue), PhD (Purdue), MASCE, FIEAust

School of Engineering, James Cook University

Townsville, Queensland, 4811, Australia

Date: 12 April 2007

Submitted to Journal of Geotechnical and Geoenvironmental Engineering, ASCE

Number of words: 9931

Number of tables: 2

Number of figures: 8

Author for correspondence:

Prof. Buddhima Indraratna

Professor of Civil Engineering

Faculty of Engineering

University of Wollongong

Wollongong City, NSW 2522

Australia

Tel: 61-2-4221 3046

Fax: 61-2-4221 3238

Email: indra@uow.edu.au 


\title{
VERTICAL AND RADIAL CONSOLIDATION ANALYSIS OF MULTI-LAYERED SOIL USING THE SPECTRAL METHOD
}

Rohan Walker ${ }^{1}$, Buddhima Indraratna ${ }^{2}$ and Nagaratnam Sivakugan ${ }^{3}$

\begin{abstract}
A new, easy to implement, solution to the consolidation of multi-layered soil based on the spectral method is presented. Combined vertical and radial drainage under instantaneous or single ramp loading is considered, ignoring well resistance. Flow in the vertical direction is based on the average hydraulic gradient at a particular depth which allows smear effects to be included. The excess pore water pressure profile across all soil layers is described by a single expression calculated with common matrix operations. Average excess pore pressures within or across any number of layers are easily calculated from the single expression. The new model is verified against other solutions from the current literature indicating that the more general spectral method model can replace the separate solutions developed for specific problems.
\end{abstract}

\footnotetext{
${ }^{1}$ Geotechnical Engineer, Coffey Geotechnics Pty Ltd \& Research Associate, Centre for Geomechanics and Railway Engineering, University of Wollongong, NSW, Australia.

2 Professor, School of Civil, Mining and Environmental Engineering, Faculty of Engineering, University of Wollongong, Wollongong City, NSW 2522, Australia

3 Associate Professor, School of Engineering, James Cook University Townsville, Queensland, 4811, Australia
} 


\section{Introduction}

Existing analytical solutions to multi-layered soil consolidation problems can be difficult to implement. The solutions, usually using the separation of variables technique, are quite lengthy involving separate general equations for each soil layer whose unknown coefficients are determined from the zeros of a transcendental equation (i.e. eigenvalues) and the relationship between boundary, interface and initial conditions. Schiffman and Stein (1970) presented equations for vertical drainage, and Horne (1964) presented equations for combined vertical and radial drainage. More recent work has developed newer techniques for modeling stratified soil. Chen et al. (2005) introduced the differential quadrature method to analyze one-dimensional consolidation of multiple soil layers. Nogami and Li (2002, 2003) use the matrix transfer method in considering radial/horizontal and vertical flow in layered soil with thin sand layers, greatly simplifying the determination of eigenvalues in the vertical direction. A number of solutions exist for two layer systems. Zhu and Yin (2005) presented design charts for vertical drainage with two layers. Xie et al. (1999) solved the same problem with partially drained boundaries, while Xie et al. (2002) incorporated small strain theory and nonlinear soil properties where the decrease in permeability is proportional to the decrease in compressibility (i.e. coefficient of consolidation is constant). Double layered ground with radial and vertical drainage was studied by Tang and Onitsuka (2001) and Wang and Jiao (2004). The two layer solutions can be used to study partially penetrating vertical drains. 
The complexity of the above analytical methods limits their use, with geotechnical engineers and researchers relying on numerical methods (such as Onoue, 1988b) or one of the many idealized single layer solutions proposed by Terzaghi (1943), Barron(1948), Yoshikuni and Nakanodo (1974), Onoue (1988a), Hansbo (1981, 2001), Tang and Onitsuka (2000), Zhu and Yin (2001), Han and Ye (2002), and Leo (2004). This paper presents a new approach to solving multi-layered soil consolidation problems that is easier to implement than the above multi-layered solutions. Combined vertical and radial drainage under instantaneous or single ramp loading is considered. The powerful spectral method (Boyd, 2000) is used to solve the governing equation, producing a single expression, calculated with common matrix operations, to give the pore pressure profile across all soil layers. The new model is verified against other solutions from the current literature.

\section{Governing differential equation}

Consider a multilayered soil cylinder with a vertical drain as shown in Figure 1. Soil properties are assumed constant within each layer and do not vary with time. Drainage conditions in the vertical direction are either, pervious top and pervious bottom (PTPB), or pervious top and impervious bottom (PTIB). The velocity of pore water flow is goverened by Darcy’s Law, however, in the vertical direction, following the approach of Tang and Onitsuka (2000) and Wang and Jiao (2004), flow is based on the average hydraulic gradient at a particular depth (i.e $\partial \bar{u} / \partial Z$ as opposed to $\partial u / \partial Z$ ). Well resistance is ignored. Loading is either instantaneous or a single ramp (see Figure 2). Hansbo's (1981) method for developing radial consolidation equations can be extended to 
derive the governing equation for consolidation by combined radial and vertical flow which is given by (see Appendix A for derivation):

$$
\frac{m_{v}}{\bar{m}_{v}} \frac{\partial \bar{u}}{\partial t}=-\left[d T_{h} \frac{\eta}{\bar{\eta}} \bar{u}-d T_{v} \frac{\partial}{\partial Z}\left(\frac{k_{v}}{\bar{k}_{v}} \frac{\partial \bar{u}}{\partial Z}\right)\right]+\frac{m_{v}}{\bar{m}_{v}} \frac{\partial \bar{\sigma}}{\partial t}
$$

where, $Z=\frac{Z}{H}, d T_{v}=\frac{\bar{c}_{v}}{H^{2}}, d T_{h}=\frac{2 \bar{\eta}}{\gamma_{w} \bar{m}_{v}}, \bar{c}_{v}=\frac{\bar{k}_{v}}{\gamma_{w} \bar{m}_{v}}, \eta=\frac{k_{h}}{r_{e}^{2} \mu}$

In the preceding $\bar{u}=$ average excess pore pressure at a particular depth, $\bar{\sigma}=$ average total stress at a particular depth, $t=$ time, $z=$ depth, $H=$ depth of soil, $\gamma_{w}=$ unit weight of water, $m_{v}=$ volume compressibility, $k_{v}=$ vertical permeability, $k_{h}=$ undisturbed horizontal permeability, $r_{e}=$ drain influence radius and $\mu=$ dimensionless drain geometry/smear zone parameter. The values of $\mu$ for no smear zone, a smear zone with constant reduced permeability (Hansbo 1981), and a smear zone with parabolically varying permeability (Walker and Indraratna, 2006) are given respectively by:

$$
\begin{gathered}
\mu=\ln (n)-0.75 \\
\mu=\ln (n / s)+\left(k_{h} / k_{h}^{\prime}\right) \ln (s)-0.75 \\
\mu=\ln \left(\frac{n}{s}\right)-\frac{3}{4}+\frac{\kappa(s-1)^{2}}{\left(s^{2}-2 \kappa s+\kappa\right)} \ln \left(\frac{s}{\sqrt{\kappa}}\right)-\frac{s(s-1) \sqrt{\kappa(\kappa-1)}}{2\left(s^{2}-2 \kappa s+\kappa\right)} \ln \left(\frac{\sqrt{\kappa}+\sqrt{\kappa-1}}{\sqrt{\kappa}-\sqrt{\kappa-1}}\right)
\end{gathered}
$$

where, $n=r_{e} / r_{w}$ and $s=r_{s} / r_{w}, r_{w}=$ drain radius, $r_{s}=$ smear zone radius, and $\kappa$ is the ratio of undisturbed horizontal permeability to smear zone permeability at the drain/soil interface.

To prevent horizontal drainage within a particular soil layer $\eta$ is set equal to zero. This is useful for analyzing problems with partially penetrating vertical drains. Soil layers 
below the penetration depth will have $\eta=0$ while still allowing vertical drainage. The present method can also predict the effect of using both long and short drains in unison. In the lower soil layers where only the longer drains are present, $\eta$ will be less than in the upper layers where both long and short PVD provide drainage.

\section{Analytical solution}

The solution of Equation (1) using the spectral method is given in matrix notation as (see Appendix B for derivation):

$$
\bar{u}(Z, t) \approx u_{0} \mathbf{\Phi v} \mathbf{E}(\boldsymbol{\Gamma v})^{-1} \boldsymbol{\theta}
$$

Each matrix in Equation (3) is now defined.

$$
\boldsymbol{\Phi}=\left[\begin{array}{llll}
\phi_{1}(z) & \phi_{2}(Z) & \ldots & \phi_{N}(Z)
\end{array}\right]
$$

where,

$$
\begin{gathered}
\phi_{j}(Z)=\sin \left(M_{j} Z\right) \\
M_{j}=\left\{\begin{array}{cc}
j \pi & \text { for PTPB } \\
\frac{\pi}{2}(2 j-1) & \text { for PTIB }
\end{array}\right.
\end{gathered}
$$

The diagonal matrix $\mathbf{E}$ (square matrix with non-diagonal terms equal to zero) has diagonal elements:

$$
E_{i i}= \begin{cases}\exp \left[-\lambda_{i} t\right] & \text { instantaneous loading } \\ \frac{\left(\exp \left[-\left(\mathrm{t}-\mathrm{t}_{\mathrm{f}}\right) \lambda_{i}\right]-\exp \left[-\mathrm{t} \lambda_{i}\right]\right)}{\lambda_{i} t_{c}} & \text { ramp loading }\end{cases}
$$

where,

$$
t_{f}=\min \left[t, t_{c}\right]
$$

$\lambda_{i}$ is the $i^{\text {th }}$ eigenvalue of the matrix $\boldsymbol{\Gamma}^{-1} \boldsymbol{\Psi}$ defined below. The eigenvector associated with each eigenvalue makes up the columns of matrix $\mathbf{v}$ (i.e. $v_{i 1}$ is the eigenvector associated with $\lambda_{1}$ ). The elements of the square matrices $\boldsymbol{\Gamma}$ and $\boldsymbol{\Psi}$ are: 


$$
\Psi_{i j}=\sum_{l=1}^{m} d T_{v} M_{j} M_{i} \Lambda_{i j}^{+} k_{v l} / \bar{k}_{v}+d T_{h} \Lambda_{i j}^{-} \eta_{l} / \bar{\eta}
$$

$$
\Gamma_{i j}=\sum_{l=1}^{m} \Lambda_{i j}^{-} m_{v l} / \bar{m}_{v}
$$

where,

$$
\begin{gathered}
\Lambda_{i j}^{ \pm}= \begin{cases}S N\left[M^{-}\right] \pm S N\left[M^{+}\right] & i \neq j \\
\left(Z_{l}-Z_{l-1}\right) \pm S N\left[M^{+}\right] & i=j\end{cases} \\
S N[\beta]=\left(\sin \left(\beta Z_{l}\right)-\sin \left(\beta Z_{l-1}\right)\right) / \beta \\
M^{+}=M_{j}+M_{i}, M^{-}=M_{j}-M_{i}
\end{gathered}
$$

$\boldsymbol{\theta}$ is a column vector defined by:

$$
\begin{gathered}
\boldsymbol{\theta}^{T}=\left[\begin{array}{llll}
\theta_{1} & \theta_{2} & \ldots & \theta_{N}
\end{array}\right] \\
\theta_{i}=2 \sum_{l=1}^{m}\left[m_{v l} / \bar{m}_{v} \operatorname{CS}\left(M_{i}\right)\right]
\end{gathered}
$$

where,

$$
C S[\beta]=\left(\cos \left(\beta Z_{l}\right)-\cos \left(\beta Z_{l-1}\right)\right) / \beta
$$

To find the average pore pressure between depth $Z_{1}$ and $Z_{2}$ the $\phi_{j}(Z)$ terms in $\boldsymbol{\Phi}$ are replaced with:

$$
\bar{\phi}_{j}\left(Z_{1}, Z_{2}\right)=-C S\left(M_{j}\right) /\left(Z_{2}-Z_{1}\right)
$$

The most difficult part of the above analysis is calculating the eigenvalues and eigenvectors of the matrix $\boldsymbol{\Gamma}^{-1} \boldsymbol{\Psi}$. However, there is a comprehensive literature on eigenproblems (Hoffman, 1992) and many software programs exist to solve them. Eigenproblems can be easily solved with freeware subroutines for MS Excel (Volpi, 2005) and Fortran (Anderson et al., 1999). Proprietary programs such as Mathematica 
(Wolfram Research, Inc., 2004) and Matlab (The MathWorks Inc., 2003) may be used as well.

Figure 3 shows a flow chart of the calculation steps involved in the above process. To improve the accuracy of the solution the number of terms in the solution, $N$, (i.e. the dimensions of the matrices) is increased. A Microsoft Excel Spreadsheet program (coded in Visual Basic for Applications (VBA) has been developed to implement the equations presented in the paper. The URL web address to this program is:

http://www.uow.edu.au/eng/research/geotechnical/software/

The spreadsheet contains the verification examples presented in the paper and the VBA code with Authors’ comments.

\section{Verification against existing solutions}

To verify the new model, the equations presented above are compared with various analytical solutions taken from the literature, as listed below.

(a) Multi-layered free strain with thin sand layers separating clay layers (Nogami and Li, 2003)

(b) Double layered ground with vertical and radial drainage (Nogami and Li, 2003)

(c) Ramp loading (Tang and Onitsuka, 2001)

(d) Partially penetrating vertical drains (Runnesson et al., 1985)

(e) Vertical consolidation of four layers (Schiffman and Stein, 1970) 
(a) Multi-layered free strain with thin sand layers separating clay layers (Nogami and Li, 2003)

Nogami and Li (2003) developed a free-strain approach for calculating the excess pore pressure distribution for multi-layered soil with both vertical and radial drainage. An example problem is presented with a soil system consisting of two identical thin sand layers (height $h_{s}$ ) separating three identical clay layers (height $h_{c}$ ). The soil surface of the top soil layer is considered as a free drainage boundary, whereas an impervious boundary is assumed at the bottom of the third subsoil layer. Soil properties are described by $k_{\text {sand }} h_{s} h_{c} / r_{e}^{2} k_{v}=5, n=20, c_{h} h_{c}^{2} / c_{v} r_{e}^{2}=1, r_{e}=0.05, c_{v}=c_{h}=1$. The average excess pore water pressures calculated with the present approach and that of Nogami and Li (2003) are compared in Figure 4 and 40 series terms were used. Both methods are in close agreement except for slight deviations in the thin sand layers at low degree of consolidation. The close agreement shows that, as for homogenous ground (Hansbo, 1981; Barron, 1948), there is little difference between free-strain and equal strain formulations. The current method does not use Bessel functions that are associated with free-strain solutions.

(b) Double layered ground with vertical and radial drainage (Nogami and Li, 2003)

Nogami and Li (2003) present the pore pressure distribution during consolidation of a soil system with vertical and radial drainage consisting of two clay layers of equal height $h$. The material properties of the two clay layers are shown in Table for the three cases (no smear). Comparisons of the pore pressure distributions calculated with each method are shown in Figure 5 and 35 series terms were used. Any small oscillations in the proposed 
model result from an insufficient number of terms in the series solution. These oscillations only occur when radial drainage dominates (i.e. $d T_{h} \gg>d T_{v}$ )

\section{(c) Ramp loading (Tang and Onitsuka, 2001)}

Tang and Onitsuka (2001) presented an analytical solution for consolidation by vertical and radial drainage (no smear) for single layer consolidation under ramp loading. The average excess pore water is calculated with the soil/drain properties: $c_{v}=c_{h}=1$, $n=16.7, H / r_{e}=2, r_{e}=0.5$. The surcharge load, initially zero, increases to unity at $d T_{h} t=0.2$. Comparisons with the present method are shown in Figure 6 and 12 series terms were used. There is no discernable difference in the solutions.

\section{(d) Partially penetrating vertical drains (Runnesson et al., 1985)}

Runesson et al. (1985) performed finite element computations for consolidation with partially penetrating vertical drains including vertical and radial drainage. One example presented is for a clay/drain system with the following properties: $h_{1} / H=0.5, n=10$, $r_{e}=1, H^{2} c_{h} / r_{e}^{2} c_{v}=100$. The degree of consolidation calculated at various depths is compared to those calculated with the present method (Figure 7) employing 35 series terms. The differences in the two solutions are acceptable given the approximate nature of the finite element solution. 
(e) Vertical consolidation of four layers (Schiffman and Stein, 1970)

Schiffman and Stein (1970) present an analytical solution for one-dimensional consolidation of a layered system. The method is illustrated with an example problem consisting of four layers draining at the top and bottom. The soil properties are given in Table . The average excess pore water pressure calculated is compared with the present method in Figure and 40 series terms were used. The differences in the model are very small. 


\section{Conclusions}

The spectral method model presented above provides a powerful tool for analyzing multilayered soil consolidation problems. The most important of the spectral methods many advantages is its ease of implementation. Given the wide availability of computer software to solve matrix eigenproblems, the most difficult step in the process (determining eigenvalues and eigenvectors of a square matrix) is straightforward. The excess pore water pressure profile across all soil layers is conveniently described by a single expression, whereas existing solutions to multi-layered consolidation problems have a separate equation for each soil layer. Calculation of average excess pore pressures within or across any number of layers is far easier with a single expression. By basing flow in the vertical direction on the average hydraulic gradient at a particular depth, smear effects, not included in existing multi-layered solutions, can be included. No new smear zone parameters are introduced as the same $\mu$ parameter used in other equal strain-analyses of vertical drain problems is reproduced. The $\mu$ parameter is part of the lumped $\eta$ parameter (see Equation 1) which when varied between layers allows analysis of partially penetrating drains, smear zone variations with depth, and drain patterns with different length drains. The spectral method model is thus a very useful, easy to implement, tool in the analysis of a wide variety of multi-layered soil consolidation problems.

\section{Acknowledgements}

The Authors gratefully acknowledge the financial assistance from Australian Research Council and Queensland Department of Main Roads during the first Author's PhD 
program at University of Wollongong. The assistance of Dr. Cholachat Rujikiatkamjorn during the preparation of the final paper is appreciated. 


\section{References}

Anderson, E., Bai, Z., Bischof, C., Blackford, S., Demmel, J., Dongarra, J., Du Croz, J., Greenbaum, A., Hammarling, S., McKenney, A. and Sorensen D. (1999). LAPACK Users' Guide. Philadelphia, Society for Industrial and Applied Mathematics.

Asmar, N.H. (2004). Partial differential equations with Fourier series and boundary value problems. Upper Saddle River, NJ, Pearson Prentice Hall.

Barron, R.A. (1948). “Consolidation of fine-grained soils by drain wells.” Transactions, ASCE, 113, 718-742.

Boyd, J. (2000). Chebyshev and Fourier spectral methods. New York, DOVER Publications, Inc.

Chen, R.P., Zhou, W.H., Wang, H.Z. and Chen, Y.M. (2005). "One-dimensional nonlinear consolidation of multi-layered soil by differential quadrature method." Computers and Geotechnics, 32, 358-369.

Han, J. and Ye, S.L. (2002). "A theoretical solution for consolidation rates of stone column-reinforced foundations accounting for smear and well resistance effects." International Journal of Geomechanics, 2(2), 135-151.

Hansbo, S. (1981). "Consolidation of fine-grained soils by prefabricated drains." Proc. 10th International Conference Soil Mechanics and Foundation Engineering, Stokholm, 677-682.

Hansbo, S. (2001). "Consolidation equation valid for both Darcian and non-Darcian flow." Geotechnique, 51(1), 51-54.

Horne, M.R. (1964). "The consolidation of a stratified soil with vertical and horizontal drainage." International Journal of Mechanics and Science, 6, 187-197. 
Leo, C.J. (2004). "Equal strain consolidation by vertical drains." Journal of Geotechnical and Geoenvironmental Engineering, ASCE, 130(3), 316-327.

Nogami, T. and Li, M. (2002). "Consolidation of system of clay and thin sand layers." Soil and Foundations, Japanese Geotechnical Society, 42(4), 1-11.

Nogami, T. and Li, M. (2003). "Consolidation of clay with a system of vertical and horizontal drains." Journal of Geotechnical and Geoenvironmental Engineering, ASCE, 129(9), 838-848.

Onoue, A. (1988a). "Consolidation by vertical drains taking well resistance and smear into consideration." Soil and Foundations, Japanese Geotechnical Society, 28(4), 165-174.

Onoue, A. (1988b). "Consolidation of multi-layered anisotropic soils by vertical drains with well resistance." Soil and Foundations, Japanese Geotechnical Society, 28(3), 75-90.

Runesson, K., Hansbo, S., and Wiberg, N.E. (1985). "The efficiency of partially penetrating vertical drains." Geotechnique, 35(4), 511-516.

Schiffman, R.L. and Stein J.R. (1970). "One-dimensional consolidation of layered systems." Journal of Soil Mechanics and Foundation Engineering, ASCE, 96(4), $1499-504$.

Tang, X.W. and Onitsuka K. (2000). "Consolidation by vertical drains under timedependent loading." International Journal for Numerical and Analytical Methods in Geomechanics, 24(9), 739-751.

Tang, X.W. and Onitsuka K. (2001). "Consolidation of double-layered ground with vertical drains." International Journal for Numerical and Analytical Methods in 
Geomechanics, 25(14), 1449-1465.

Terzaghi, K. (1943). Theoretical Soil Mechanics. New York, John Wiley and Sons.

The MathWorks Inc. (2003). MATLAB. Natick, Massachusetts, The MathWorks Inc.

Volpi, L. (2005). MATRIX - Matrix and Linear Algebra addin for EXCEL. Piombini, Italy, Foxes Team.

Walker, R. and Indraratna B. (2006). "Vertical drain consolidation with parabolic distribution of permeability in smear zone." Journal of Geotechnical and Geoenvironmental Engineering, ASCE, 132(7), 937-941.

Wang, X.-S. and Jiao J.J. (2004). "Analysis of soil consolidation by vertical drains with double porosity model." International Journal for Numerical and Analytical Methods in Geomechanics, 28, 1385-1400.

Wolfram Research Inc. (2004). MATHEMATICA. Illinois, Wolfram Research, Inc.

Xie, K.-H., Xie, X.-Y. and Gao X. (1999). "Theory of one dimensional consolidation of two-layered soil with partially drained boundaries." Computers and Geotechnics, 24, 265-278.

Xie, K.-H., Xie, X.-Y. and Jiang, W. (2002). "A study on one-dimensional nonlinear consolidation of double-layered soil." Computers and Geotechnics, 29, 151-168.

Yoshikuni, H. and Nakanodo, H. (1974). "Consolidation of Fine-Grained Soils by Drain Wells with Finite Permeability." Soil and Foundations, Japanese Geotechnical Society, 14(2), 35-46.

Zhu, G. and Yin, J.-H. (2005). "Solution charts for the consolidation of double soil layers." Canadian Geotechnical Journal, 42, 949-956. 


\section{Appendix A: Derivation of governing equation}

For the unit cell in Figure 1, the velocity of flow in the radial direction, $v_{r}$, is described by Darcy's law:

$$
v_{r}=-\frac{k_{h}}{\gamma_{w}} \frac{\partial u}{\partial r}
$$

In the vertical direction, following the approach of Tang and Onitsuka (2000) and Wang and Jiao (2004), Darcy's law is modified to include the average excess pore water pressure at a particular depth, $\bar{u}$. The velocity of flow in the vertical direction, $v_{v}$, is then given by:

$$
v_{v}=-\frac{k_{v}}{\gamma_{w}} \frac{\partial \bar{u}}{\partial z}
$$

Deformation is assumed to take place solely in the vertical direction under equal-strain conditions (Barron, 1948; Hansbo, 1981), hence:

$$
\frac{\partial \varepsilon}{\partial t}=m_{v}\left(\frac{\partial \bar{\sigma}}{\partial t}-\frac{\partial \bar{u}}{\partial t}\right)
$$

where, $\partial \varepsilon / \partial t=$ vertical strain rate. Following Hansbo’s (1981) approach (modified to include vertical drainage), flow into and out of a cylindrical slice with internal radius $r$, and external radius $r_{e}$ is considered. The resulting expressions for pore water pressure gradient is (ignoring smear effects):

$$
\frac{\partial u}{\partial r}=\frac{\gamma_{w}}{2 k_{h}}\left(\frac{r_{e}^{2}}{r}-r\right)\left[\frac{\bar{k}_{v}}{\gamma_{w} H^{2}} \frac{\partial}{\partial Z}\left(\frac{k_{v}}{\bar{k}_{v}} \frac{\partial \bar{u}}{\partial Z}\right)+\frac{\partial \varepsilon}{\partial t}\right]
$$

The average excess pore pressure in the soil cylinder at depth $Z$ is found from the following algebraic expression: 


$$
\bar{u} \pi\left(r_{e}^{2}-r_{w}^{2}\right)=\int_{r_{w}}^{r_{e}} 2 \pi r u d r
$$

Equation (A4) is integrated in the $r$ direction (noting $k_{v}, m_{v}$, and $\sigma$ are independent of $r$ ) with the boundary condition $u\left(r_{w}, t\right)=0$ (i.e. well resistance and vacuum loading are not considered). The resulting expression combined with Equations (A3) and (A5) give the average pore water pressure at normalized depth $Z$ :

$$
\bar{u}=\frac{r_{e}^{2} \gamma_{w} \mu}{2 k_{h}}\left[\frac{\bar{k}_{v}}{\gamma_{w} H^{2}} \frac{\partial}{\partial Z}\left(\frac{k_{v}}{\bar{k}_{v}} \frac{\partial \bar{u}}{\partial Z}\right)+\bar{m}_{v} \frac{m_{v}}{\bar{m}_{v}}\left(\frac{\partial \bar{\sigma}}{\partial t}-\frac{\partial \bar{u}}{\partial t}\right)\right]
$$

The $\mu$ parameter is that of Equation (2a). Equation (A6) can be derived for various smear zone properties, in which case only the $\mu$ parameter will differ as in Equations (2b) and (2c). Using the definitions of $\eta, c_{v}, d T_{h}$ and $d T_{v}$ form Equation (1), Equation (A6) is now rearranged to give the governing differential equation:

$$
\frac{m_{v}}{\bar{m}_{v}} \frac{\partial \bar{u}}{\partial t}=-\left[d T_{h} \frac{\eta}{\bar{\eta}} \bar{u}-d T_{v} \frac{\partial}{\partial Z}\left(\frac{k_{v}}{\bar{k}_{v}} \frac{\partial \bar{u}}{\partial Z}\right)\right]+\frac{m_{v}}{\bar{m}_{v}} \frac{\partial \bar{\sigma}}{\partial t}
$$

The solution of Equation (A7) by the spectral method (Boyd, 2000) is developed in Appendix B. 


\section{Appendix B: Solution of governing equation by spectral method}

Equation (A7) is a non-homogeneous partial differential equation with source/sink terms.

The source/sink term, $f(Z, t)$, is a function of depth and time, and arises from surcharge:

$$
f(Z, t)=\frac{m_{v}}{\bar{m}_{v}} \frac{\partial \bar{\sigma}}{\partial t}
$$

To solve Equation (A7) Duhamel's principle (Asmar, 2004) is used: $f(Z, t)$ is replaced by an impulse load applied at time $\tau$ and depth $\zeta$, and a 'fundamental solution', $\bar{u}_{\delta}(Z, t, \zeta, \tau)$, is obtained (with the initial condition $u(Z, 0)=0$ ). The impulse load is then described by:

$$
f(Z, t)=\delta(Z-\zeta) \delta(t-\tau)
$$

where, $\delta(x)$ is the Dirac Delta function. Once the fundamental solution is known, the complete solution is given by:

$$
\bar{u}(Z, t)=\int_{0}^{\tau} \int_{0}^{1} \bar{u}_{\delta}(Z, t, \zeta, \tau) f(Z, t) d \zeta d \tau
$$

A partial differential equation such as Equation (A7) can be expressed in a shorthand form as:

$$
L(\bar{u}(Z, t))=f(Z, t)
$$

where, $L$ is an operator involving partial derivatives. The spectral method involves expressing $\bar{u}(Z, t)$ as a truncated series of $N$ terms which in matrix form is:

$$
\bar{u}(Z, t) \approx \mathbf{\Phi} \mathbf{A}
$$


In matrix $\boldsymbol{\Phi}, \phi_{j}(Z)$ which area set of linearly independent basis-functions, and in matrix A, $A_{j}(t)$ are unknown coefficients. The basis functions are generally chosen to satisfy the boundary conditions. For pervious top and pervious bottom (PTPB) $\bar{u}(0, t)=0$ and $\bar{u}(H, t)=0$, and for pervious top and impervious bottom (PTIB) $\bar{u}(0, t)=0$ and $\partial \bar{u}(H, t) / \partial z=0$. Suitable basis functions are those given in Equation (4b).

The error, $e_{r}$, of Equation (B5) that satisfies Equation (B4) is

$$
e_{r} \approx L(\mathbf{\Phi} \mathbf{A})-f(Z, t)
$$

The Galerkin procedure requires that the error be orthogonal to each basis function, hence:

$$
\int_{0}^{1} e_{r} \phi_{i} d Z=0, \text { for } i=1, \ldots, N
$$

Substituting Equation (B6) into Equation (B7a) yields:

$$
\int_{0}^{1} \phi_{i} L(\mathbf{\Phi A}) d Z-\int_{0}^{1} \phi_{i} f(Z, t) d Z=0
$$

which is a set of coupled ordinary differential equations for $\mathrm{A}_{j}$. Substituting Equations (A7), (B2), (B4), and (B5) into Equation (B7) and integrating gives the matrix equations:

$$
\mathbf{\Gamma} \mathbf{A}^{\prime}=-\boldsymbol{\Psi} \mathbf{A}+\boldsymbol{\Phi}(\zeta)^{T} \delta(t-\tau)
$$

where,

$$
\mathbf{A}^{\prime}=\frac{\partial}{\partial t} \mathbf{A}
$$


The $m_{v}$ values of each soil layer contribute to the $\boldsymbol{\Gamma}$ matrix when the left hand side of Equation (A7) is integrated over the entire soil depth. The $k_{v}$ and $\eta$ values of each soil layer contribute to the $\boldsymbol{\Psi}$ matrix when the right hand side of Equation (A7) is integrated. $\boldsymbol{\Gamma}$ and $\boldsymbol{\Psi}$ are defined by:

$$
\begin{gathered}
\Gamma_{i j}=\int_{0}^{1} \frac{m_{v}}{\bar{m}_{v}} \phi_{i} \phi_{j} d Z \\
\boldsymbol{\Psi}_{i j}=\int_{0}^{1} d T_{h} \frac{\eta}{\bar{\eta}} \phi_{j} \phi_{i} d Z-\int_{0}^{1} d T_{v} \frac{\partial}{\partial Z}\left(\frac{k_{v}}{\bar{k}_{v}} \phi_{j}^{\prime}\right) \phi_{i} d Z
\end{gathered}
$$

For the case of constant soil properties within a layer presented in this paper each of the three parameters, $k_{v} / \bar{k}_{v}, m_{v} / \bar{m}_{v}$ and $\eta / \bar{\eta}$ are defined with the unit step function:

$$
\alpha(Z)=\alpha_{l} \operatorname{UnitStep}\left(Z-Z_{l-1}\right) \operatorname{UnitStep}\left(Z_{l}-Z\right)
$$

The derivative of the unit step function, required when differentiating $k_{v} / \bar{k}_{v}$ in Equation (B11), is the impulse or Dirac delta function. Layer interfaces are included by simply integrating across these impulse functions at each layer boundary.

Using variation of parameters (also called variation of constants), the solution to the nonhomogeneous Equation (B8) can be found using the initial condition $\mathbf{A}(0)=0$ :

$$
\begin{aligned}
\mathbf{A}(t) & =\mathbf{v E}(t)(\mathbf{v E}(0))^{-1} \mathbf{A}(0)+\mathbf{v E}(t) \int_{0}^{t}(\mathbf{v E}(y))^{-1} \boldsymbol{\Gamma}^{-1} \mathbf{\Phi}(\zeta)^{T} \delta(y-\tau) d y \\
& =\mathbf{v E}(t-\tau)(\boldsymbol{\Gamma} \mathbf{v})^{-1} \mathbf{\Phi}(\zeta)^{T}
\end{aligned}
$$

where $\mathbf{E}$ is defined by Equation (5). The fundamental solution to Equation (A7) with impulse loading is now given by: 


$$
\bar{u}_{\delta}(Z, t)=\boldsymbol{\Phi v} \mathbf{E}(t-\tau)(\Gamma \mathbf{v})^{-1} \mathbf{\Phi}(\zeta)^{T}
$$

Equation (B3) now becomes:

$$
\bar{u}(Z, t)=\int_{0}^{\tau} \int_{0}^{1} \boldsymbol{\Phi} \mathbf{v E}(t-\tau)(\boldsymbol{\Gamma v})^{-1} \mathbf{\Phi}(\zeta)^{T}\left(\frac{m_{v}}{\bar{m}_{v}} \frac{\partial \bar{\sigma}}{\partial t}\right) d \zeta d \tau
$$

The surcharge loading term in Equation (B15) can be any function of time and depth, $\bar{\sigma}(Z, t)$. For a load that is constant with depth, $\bar{\sigma}(Z, t)$ is expressed as:

$$
\bar{\sigma}(Z, t)=\left\{\begin{array}{cc}
u_{0} \operatorname{UnitStep}(t) & \text { instantaneous loading } \\
u_{0} t_{f} / t_{c} & \text { ramp loading }
\end{array}\right.
$$

where $t_{f}=\min \left[t, t_{c}\right]$. Substituting Equation (B16) into Equation (B15) results in the simple matrix expression of Equation (3). 
Table 1 Parameters for double layered ground (adopted from Nogami and Li, 2003)

\begin{tabular}{ccccccccc}
\hline Case & $r_{w}$ & $n$ & $H / r_{w}$ & $h_{2} / h_{1}$ & $k_{v 2} / k_{v 1}$ & $c_{v 2}, c_{v 1}$ & $c_{h 1}$ & $c_{h 2}$ \\
\hline 1 & 0.05 & 10 & 200 & 1 & 2 & 1 & 1 & 5 \\
2 & 0.05 & 10 & 200 & 1 & 2 & 1 & 1 & 1 \\
3 & 0.05 & 10 & 200 & 1 & 2 & 1 & 5 & 1 \\
\hline
\end{tabular}


Table 2 Soil profile, four layer system (adopted from Schiffman and Stein, 1970)

\begin{tabular}{cccc}
\hline Layer & Depth $(\mathrm{m})$ & $k_{v}(\mathrm{~m} / \mathrm{s})$ & $m_{v}\left(\mathrm{~m}^{2} / \mathrm{kN}\right)$ \\
\hline 1 & 0 to 3.05 & $2.78 \times 10^{-11}$ & $6.41 \times 10^{-5}$ \\
2 & 3.05 to 9.14 & $8.26 \times 10^{-11}$ & $4.07 \times 10^{-5}$ \\
3 & 9.14 to 18.29 & $1.17 \times 10^{-11}$ & $2.03 \times 10^{-5}$ \\
4 & 18.29 to 24.38 & $2.94 \times 10^{-11}$ & $4.07 \times 10^{-5}$ \\
\hline
\end{tabular}




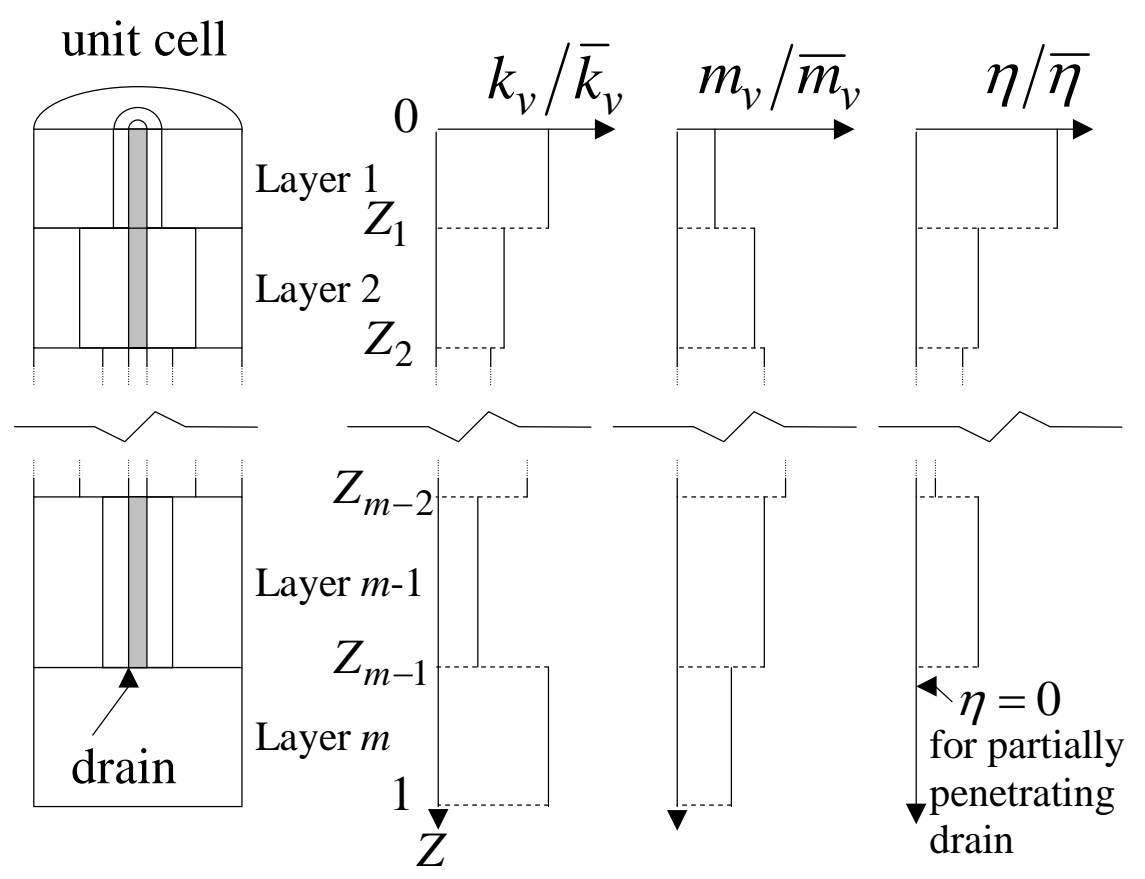

Figure 1 Multilayered soil properties 


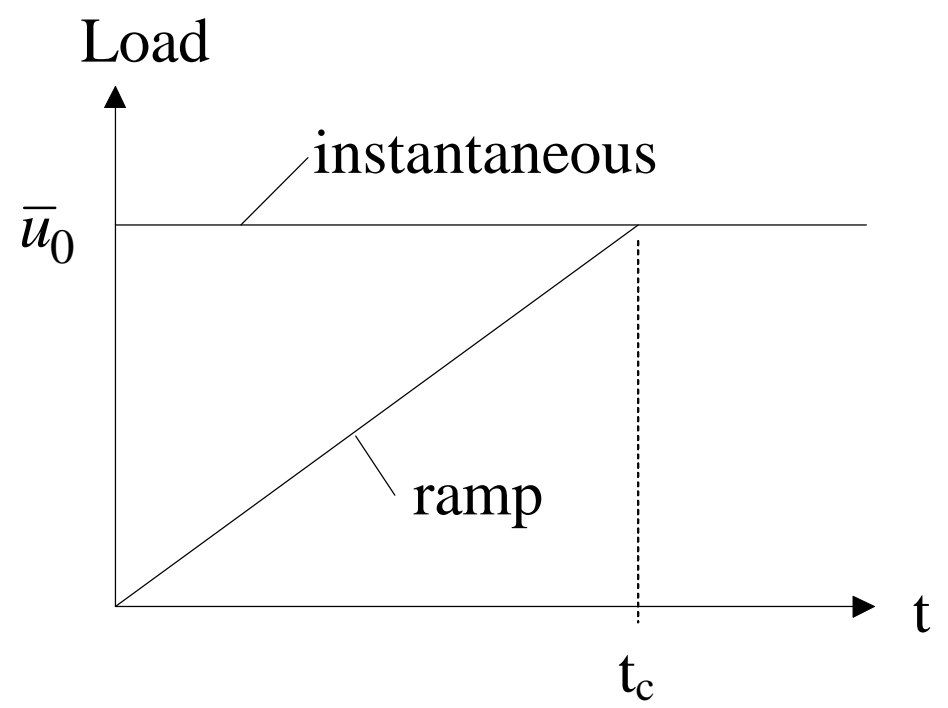

Figure 2 Instantaneous and ramp loading 


\begin{tabular}{|c|}
\hline START \\
\hline Choose soil and drainage properties \\
\hline Choose number of terms $(N)$ \\
\hline Populate $\boldsymbol{\Gamma}$ and $\boldsymbol{\Psi}$, Eqs. (6) and (7) \\
\hline Find eigenvalues $(\lambda)$ and eigenvectors ( $\mathbf{v})$ \\
\hline of $\boldsymbol{\Gamma}^{-1} \boldsymbol{\Psi}$ \\
\hline Populate $\boldsymbol{\Phi}, \mathbf{E}$ and $\boldsymbol{\theta}$, Eqs. (4), (5) and (11) \\
\hline Calculate $\bar{u}(Z, t) \approx u_{0} \mathbf{\Phi v E}(\boldsymbol{\Gamma} \mathbf{v})^{-1} \boldsymbol{\theta}$, Eq. (3) \\
\hline END \\
\hline
\end{tabular}

Figure 3 Flow chart for calculation steps 


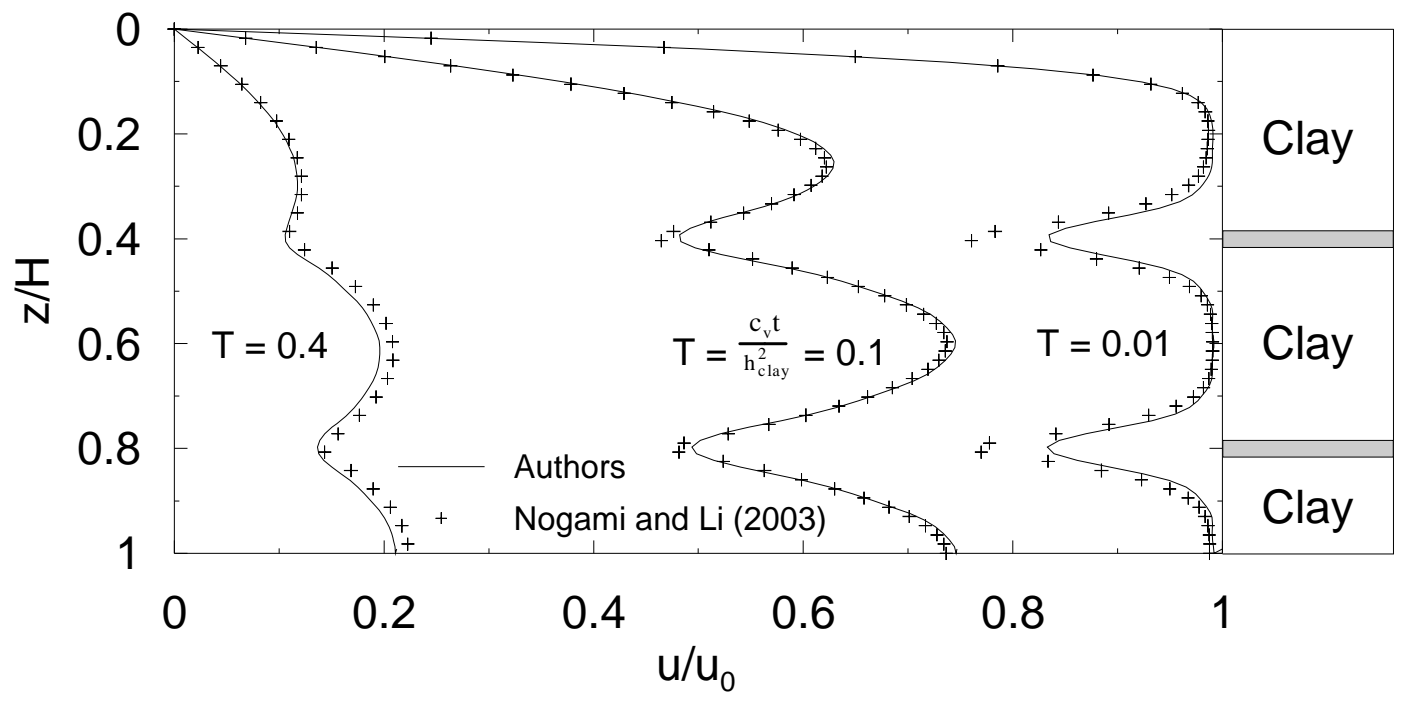

Figure 4 Model verification: multi-layer equal-strain vs free-strain 


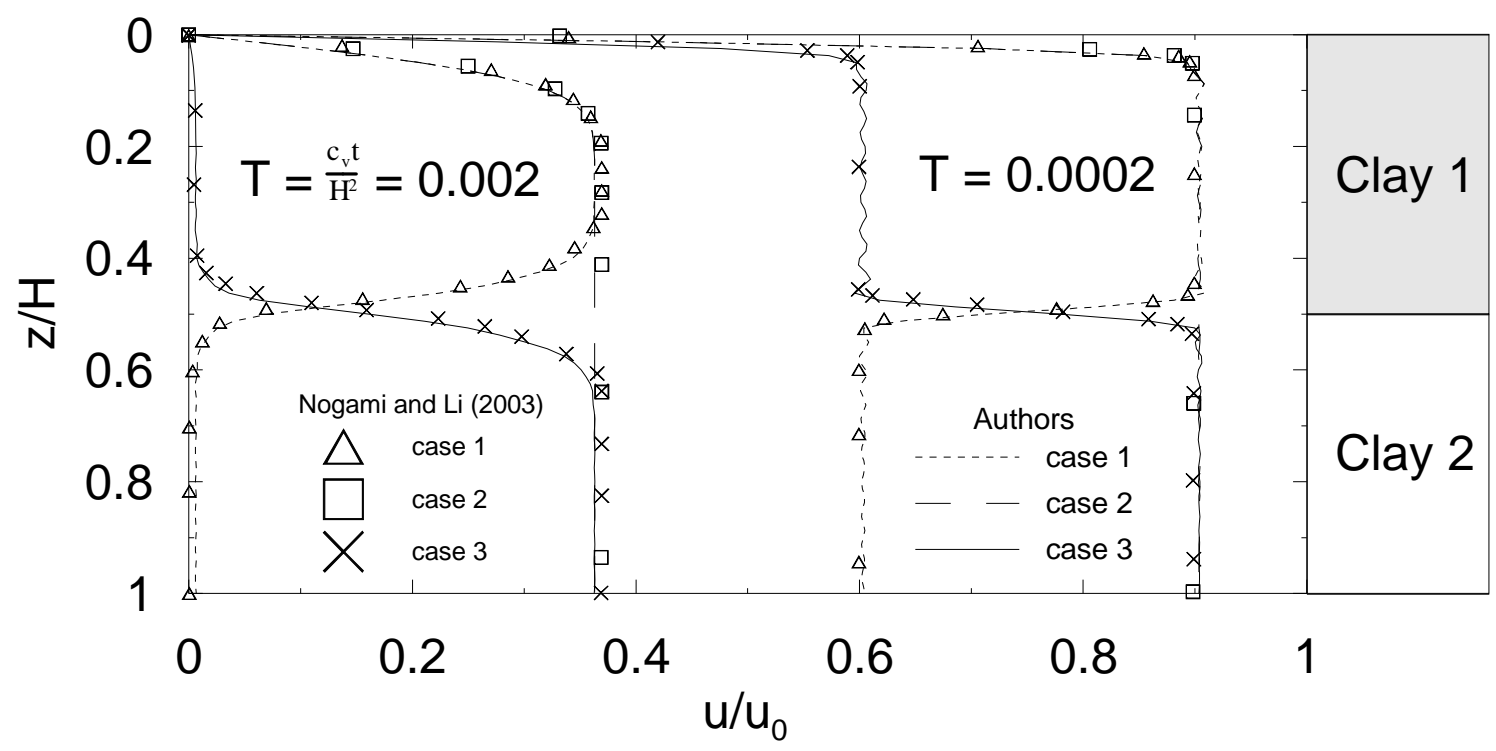

Figure 5 Model verification: double layered ground 


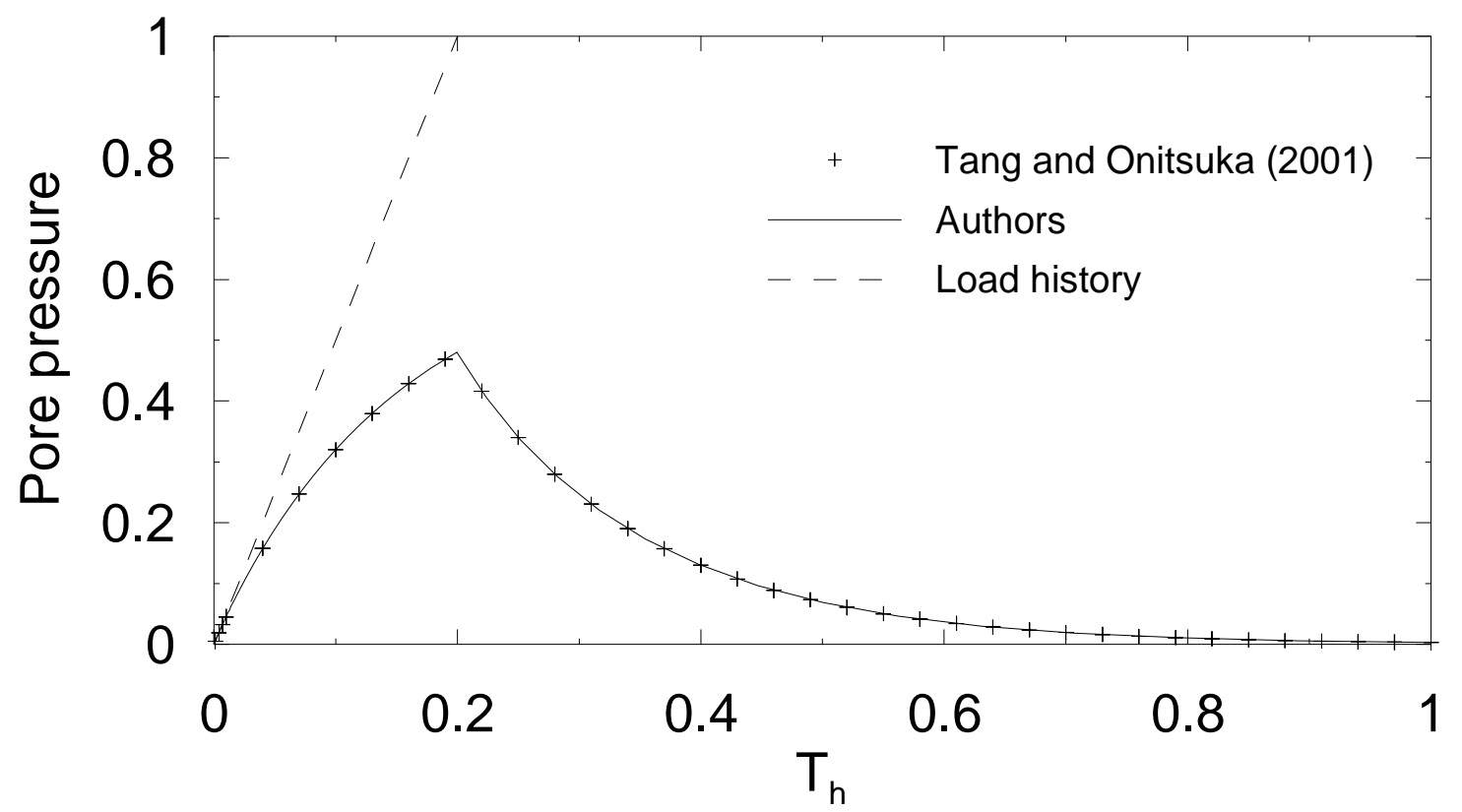

Figure 6 Model verification: multiple stage loading 


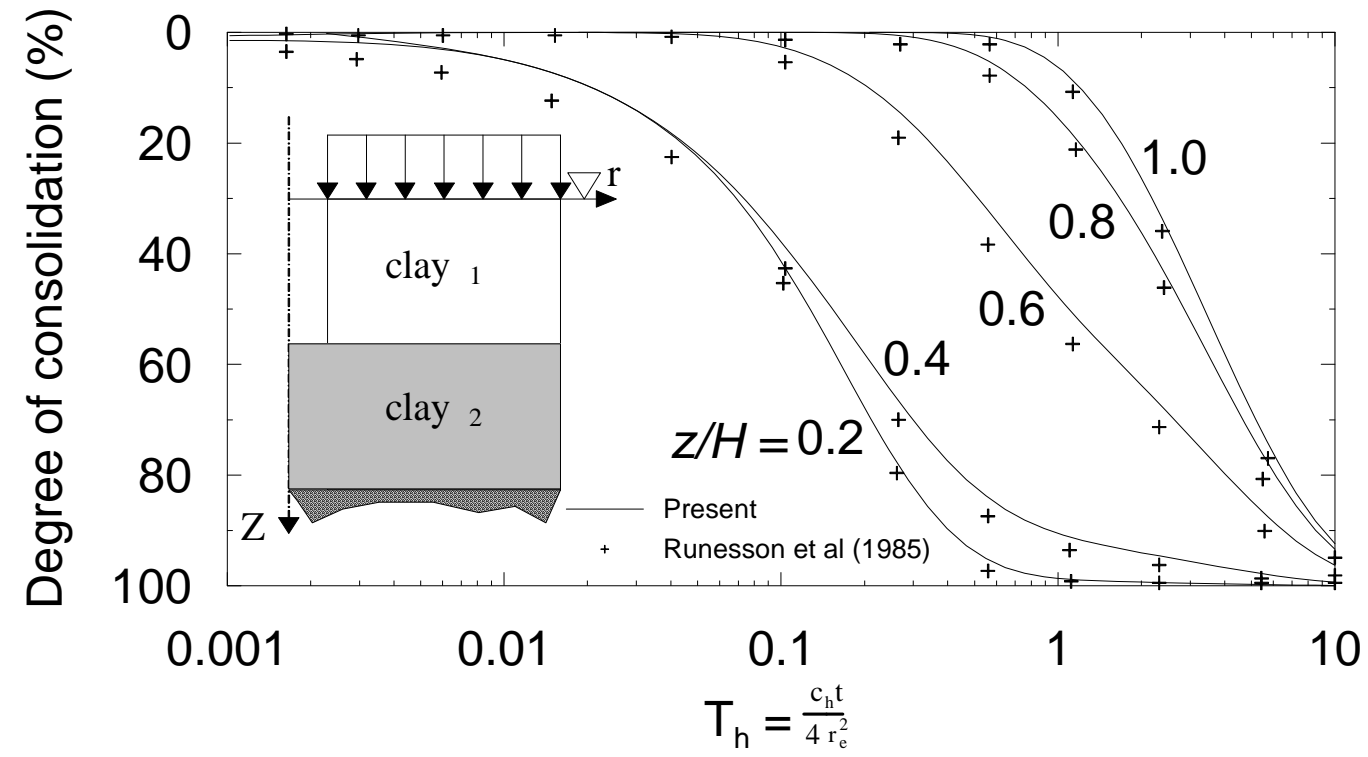

Figure 7 Model verification: partially penetrating vertical drains 


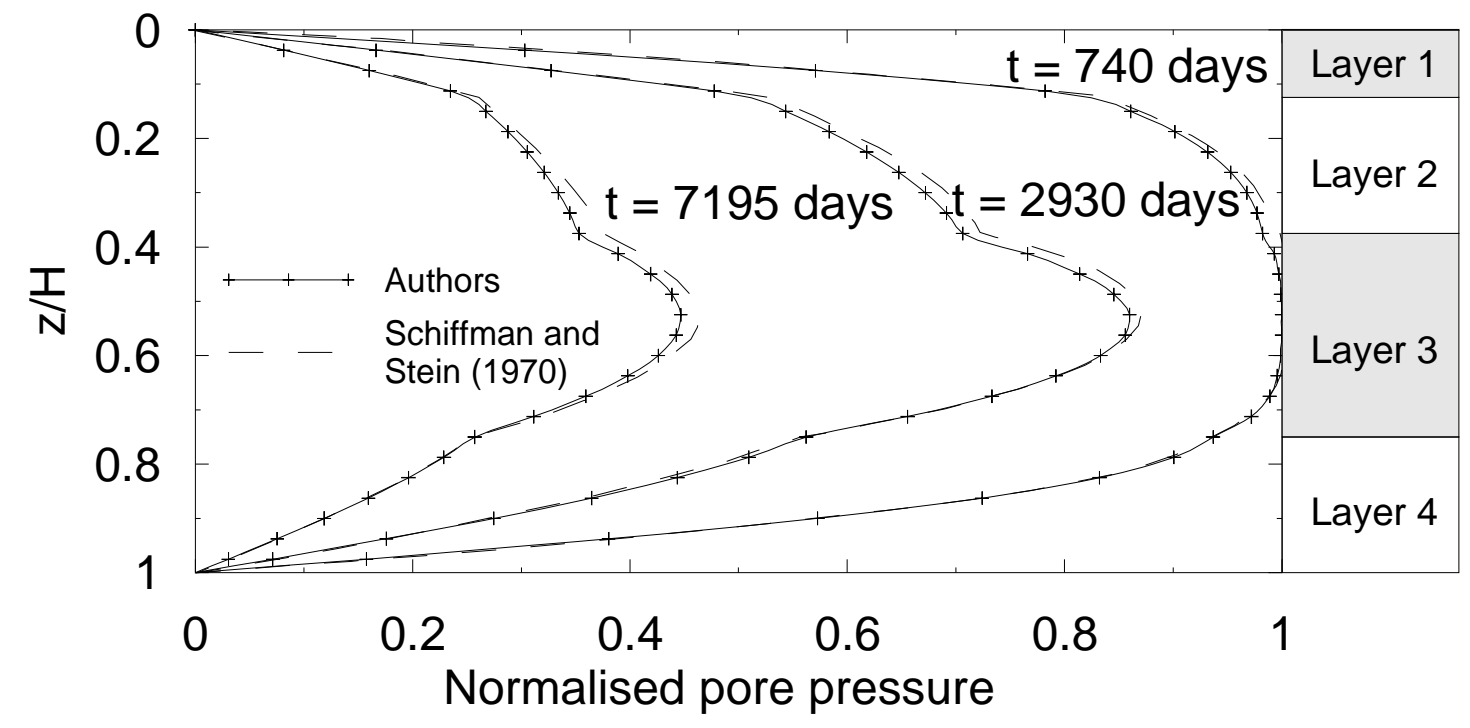

Figure 8 Model verification: 4 layer vertical drainage 